\title{
On the tungsten single crystal coatings achieved by chemical vapor transportation deposition
}

\author{
J.Q. Shi ${ }^{1}$, Y.B. Shen ${ }^{1}$, S.Y. Yao ${ }^{1}$, P.J. Zhang ${ }^{1}$, Q. Zhou ${ }^{1}$, Y.Z. Guo ${ }^{1}$, C.W. Tan ${ }^{1,2 *}$, \\ X.D. $\mathrm{Yu}^{1,2}$, Z.H. $\mathrm{Nie}^{1}$, H.L. $\mathrm{Ma}^{2}$, H.N. Cai ${ }^{1}$ \\ ${ }^{1}$ School of Materials Science and Engineering, Beijing Institute of Technology, Beijing \\ 100081, China \\ ${ }^{2}$ China Astronaut Research and Training Center, Beijing 100094, China
}

\section{Corresponding author:}

Name: Chengwen Tan

Tel: +861068912712

Fax: +861062890250

E-mail: tanchengwen@bit.edu.cn

\begin{abstract}
The tungsten single crystal has many excellent properties, namely a high melting point, high anti-creeping strength. Chemical vapor transportation deposition (CVTD) is a possible approach to achieve large-sized W single crystals for high-temperature application such as the cathode of a thermionic energy converter. In this work, CVTD
\end{abstract} 1 
W coatings were deposited on the monocrystalline molybdenum substrate (a tube with <111> axial crystalline orientation) using $\mathrm{WCl}_{6}$ as a transport medium. The microstructures of the coatings were investigated by a scanning electron microscope (SEM) and electron backscatter diffraction (EBSD). The as-deposited coatings are hexagonal prisms--rough surfaces perpendicular to $\langle 110\rangle$ with alternating hill-like bulges and pits at the side edges of the prisms, and flat surfaces perpendicular to $<112>$ with arc-shaped terraces at the side faces. This can be explained by the two-dimensional nucleation -mediated lateral growth model. Some parts of the coatings contain hillocks of an exotic morphology (noted as "abnormal growth"). The authors hypothesize that the abnormal growth is likely caused by the defects of the Mo substrate, which facilitate W nucleation sites, cause orientation difference, and may even form boundaries in the coatings. A dislocation density of $10^{6}$ to $10^{7}$ (counts $/ \mathrm{cm}^{2}$ ) was revealed by an etch-pit method and the synchrotron X-ray diffraction. As the depositing temperature rises, the dislocation density decreases, and no sub-boundaries are found on samples deposited over $1300^{\circ} \mathrm{C}$, as a result of the atom diffusion and the dislocation climbing.

Keywords: tungsten single crystal; chemical vapor transportation deposition; microstructures.

\section{Introduction}

Serving as a power source in satellites and space stations, thermionic reactors have several advantages over solar arrays commonly utilized in spacecraft, including a simple 
but stable structure with no rotating components, the possibility of miniaturization, utility in the dark, and a higher power density during constant high-power output $[1,2]$. Nevertheless, the cathode of the thermionic energy converter, which is the "heart" of the reactor, must operate at a high ambient temperature of about $1500^{\circ} \mathrm{C}$ throughout the service period. The tungsten single crystal has a combination of many excellent properties including a high melting point, high anti-creeping strength, high elastic modulus, high resistance to radiation, and a good thermionic emission work function [3, 4]. These make $\mathrm{W}$ single crystals an ideal material for the functional layers of the cathode. The microstructures of the single crystal significantly affect the lifespan and stability of the W functional layers. This is especially true when the crystal consists of many sub-grains [5]. Thus, this investigation into microstructures is important. In previous studies, electron beam floating zone melting (EBFZM) can produce bulk single crystals of high-purity $\mathrm{W}$, but not $\mathrm{W}$ single crystal coatings [6, 7]. Conventional chemical vapor deposition (CVD) of W single crystal coatings has been reported as well. Researchers have deposited thin films of $\mathrm{W}$ single crystals on $\mathrm{Si}$ and $\mathrm{Cu}$ substrates using $\mathrm{W}(\mathrm{CO})_{6}$ as a precursor, but the film contains impurity elements ( $\mathrm{C}$ and $\left.\mathrm{O}\right)$ [8-10]. Researchers of Auburn University and Uppsala University have used laser chemical vapor deposition to grow micron-sized tungsten single crystals from a reaction gas mixture of $\mathrm{WF}_{6}$ and $\mathrm{H}_{2}$ [11]. Moreover, these CVD methods usually have challenges in the preparation of large-sized $\mathrm{W}$ single crystal coatings - this limits the application of 
these methods. Chemical vapor transportation deposition (CVTD) is an emerging method for obtaining $\mathrm{W}$ single crystals. Achieving $\mathrm{W}$ single crystals by CVTD has made some substantial developments [12]. In our previous work, we have studied the CVTD of $\mathrm{W}$ on the molybdenum substrate using $\mathrm{WCl}_{6}$ as a transport medium, the proportion quantitative analysis and etching of the $\{110\}$ planes of the as-deposited $\mathrm{W}$ coatings [13-15].

Here, we studied the growth habit, abnormal growth, and defects (dislocations and sub-boundaries) of the $\mathrm{W}$ coatings grown by CVTD. The growth habit was revealed based on the W coatings' morphology. The abnormal growth on the surface of the W coatings was investigated by scanning electron microscope (SEM) and electronic backscatter diffraction (EBSD). We discuss a possible explanation for the abnormal growth. The dislocations and sub-boundaries in the coatings were studied via an etch-pit method and synchrotron X-ray diffraction.

\section{Materials and experimental}

In this work, $\mathrm{W}$ coatings were fabricated by CVTD. A polycrystalline $\mathrm{W}$ tube of high purity (total impurity content is $0.0052 \mathrm{wt} \%$ ) was used as the source of $\mathrm{W}$, and $\mathrm{WCl}_{6}$ of high purity (over 99.99\%) was used as the transport medium of $\mathrm{W}$. The monocrystalline Mo tube with $\langle 111\rangle$ crystalline orientation (crystal axial declination angle less than $3^{\circ}$ ) 
was fabricated by the Northwest Institute for Non-ferrous Metal Research using electron beam floating zone melting (EBFZM). Its dislocation density was as high as $10^{6}$ $\left(\right.$ counts $/ \mathrm{cm}^{2}$ ). The Mo tube (with the appearance of sub-boundaries) served as a substrate for the deposition of W. Mo and W have similar linear expansion coefficients and a body-centered cubic space lattice with similar lattice constants (see Table 1). Thus, cracks and thermal deformation are not likely to happen when heated - the lattice mismatch is relatively low (only $0.633 \%$ ). Three $\mathrm{W}$ coatings were prepared under different substrate temperatures. Sample $\mathrm{W}_{1300}$ was deposited at $1300^{\circ}$ C. Sample $\mathrm{W}_{1300-1400}$ was first deposited at $1300^{\circ} \mathrm{C}$ for $20 \mathrm{~min}$ and then heated to $1400^{\circ} \mathrm{C}$, and $\mathrm{W}_{1400}$ was deposited at $1400^{\circ} \mathrm{C}$. The pressure of the reaction chamber was $15.77 \mathrm{~Pa}$, the temperature of the $\mathrm{W}$ source was kept at $360^{\circ} \mathrm{C}$ below the substrate temperature, and the temperature of the evaporating chamber was $120^{\circ} \mathrm{C}$.

Table 1. Physical properties of W and Mo.

$\begin{array}{llllll}\text { Material } & \begin{array}{l}\text { Melting } \\ \text { point }\left({ }^{\circ} \mathrm{C}\right)\end{array} & \begin{array}{l}\text { Density } \\ \left(\mathbf{g} / \mathbf{c m}^{3}\right)\end{array} & \begin{array}{l}\text { Thermal } \\ \text { conductivity } \\ \left(\mathbf{c a l} /\left(\mathbf{c m} \cdot \mathbf{s}^{\circ}{ }^{\circ} \mathrm{C}\right)\right)\end{array} & \begin{array}{l}\text { Coefficient } \\ \text { of expansion } \\ \left(\mathbf{1 0}^{-6} /{ }^{\circ} \mathrm{C}\right)\end{array} & \begin{array}{l}\text { Lattice } \\ \text { constant } \\ (\AA)\end{array} \\ \text { Mo } & 2625 & 10.22 & 0.340 & 4.9 & 3.14 \\ \text { W } & 3410 & 19.30 & 0.397 & 4.6 & 3.16\end{array}$

The coatings were examined by the unaided eye and SEM (JEOL JSM-7001F thermal field emission SEM). The crystal orientations of different $\mathrm{W}$ coating surfaces were analyzed by EBSD (JEOL JSM-7001F thermal field emission SEM, with Data 
Collection 5 software developed by EDAX). The sample was placed in the sample chamber of the SEM. It was inclined at a large angle of $65^{\circ} \sim 70^{\circ}$. The CCD camera captured the Kikuchi pattern generated by electron diffraction. This was automatically analyzed by computer, after which the software generated an EBSD grain orientation map.

Although transmission electron microscope (TEM) can resolve the detail of the dislocations and other defects in the crystal, it can only observe an extremely small area each time. Rather, we used an etch-pit method in which the density of the etch-pits represents the density of the dislocations to investigate the coatings [16]. The lattice distortion caused by the presence of a dislocation allows foreign atoms to accumulate and results in a relatively higher energy state in the atoms adjacent to the dislocation. The dislocation outcrop is therefore more easily attacked due to foreign atoms and the higher energy state. It makes the etch-pit visible with microscopy under appropriate etching conditions. After repeated experiments, we found a feasible etchant that used a solution of $10 \mathrm{~g} \mathrm{NaOH}$ and $10 \mathrm{~g} \mathrm{~K}_{3} \mathrm{Fe}(\mathrm{CN})_{6}$ dissolved in $80 \mathrm{ml}$ deionized water. The etch-pits obtained could be well distinguished and facilitated automatic computer-based analysis of dislocation density. After being ground by abrasive paper and polished to a fine finish, the $\mathrm{W}$ coatings were etched and observed under an optical microscope (OM, OLYMPUS BX51M). The OLYCIA M3 - a metal analysis software-was used to 
calculate the dislocation density of the samples.

Fig. 1 shows three typical types of different etch-pit morphologies etched by the etchant mentioned above. As shown in the figure, the etch-pit of the $\{110\}$ planes are an incomplete rhombus (Fig. 1a). For the $\{112\}$ planes, it is an irregular quadrilateral (Fig. 1b). For the $\{111\}$ planes, it is an equilateral triangle (Fig. 1c).

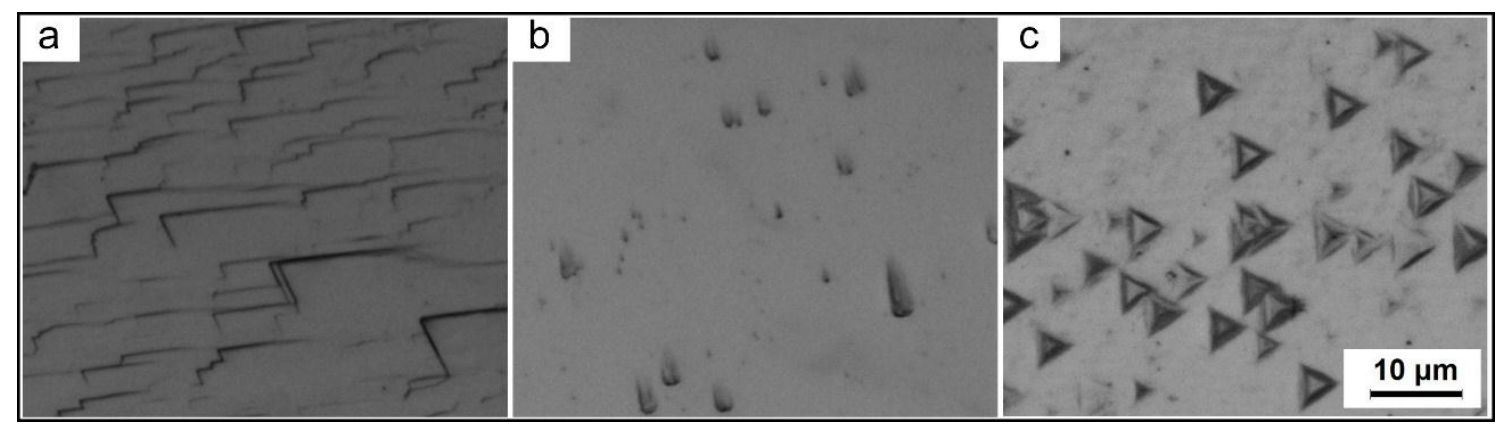

Fig. 1 Three typical types of different etch-pit morphologies, (a) $\{110\}$, (b) $\{112\}$ and (c) $\{111\}$.

The formation of different etch-pit morphologies can be explained by the following theory. The W has a BCC structure and the close-packed $\{110\}$ planes show the lowest surface energy $[17,18]$. After etching, the $\{110\}$ planes will eventually be exposed to show intersections of the surface planes and $\{110\}$ planes form the shapes of these etch-pits. According to the zone law, let $\mathrm{R}_{\mathrm{i}}$ be the vector of the intersection of plane $\mathrm{H}$ and plane $\mathrm{A}_{\mathrm{i}}$. Then, $R_{i}=H \times A_{i}$. And let $\phi_{\mathrm{ij}}$ be the angle between $\mathrm{Ri}$ and $\mathrm{R}_{\mathrm{j}}$, and then $\cos \theta_{i j}=\left(R_{i} \cdot R_{j}\right) /\left(\left|R_{i}\right| \cdot\left|R_{j}\right|\right) . \quad$ Take the (111) plane for example. 


$$
R_{(110)}=[111] \times[110]=\left[\begin{array}{lll}
i & j & k \\
1 & 1 & 1 \\
1 & 1 & 0
\end{array}\right]=[\overline{1} 10]
$$

By applying the same mathematical technique, we can show that the intersections of the

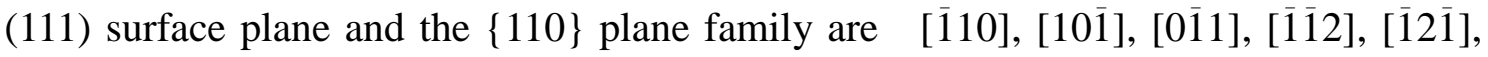
and $[2 \overline{1} \overline{1}]$. The angles between them are $30^{\circ}, 60^{\circ}, 90^{\circ}, 120^{\circ}$ and $150^{\circ}$. Thus, we can obtain a geometric model of the etch-pit of (111) (shown in Fig. 2a). It is the same for the (112) plane (see Fig. 2b) and the (110) plane.

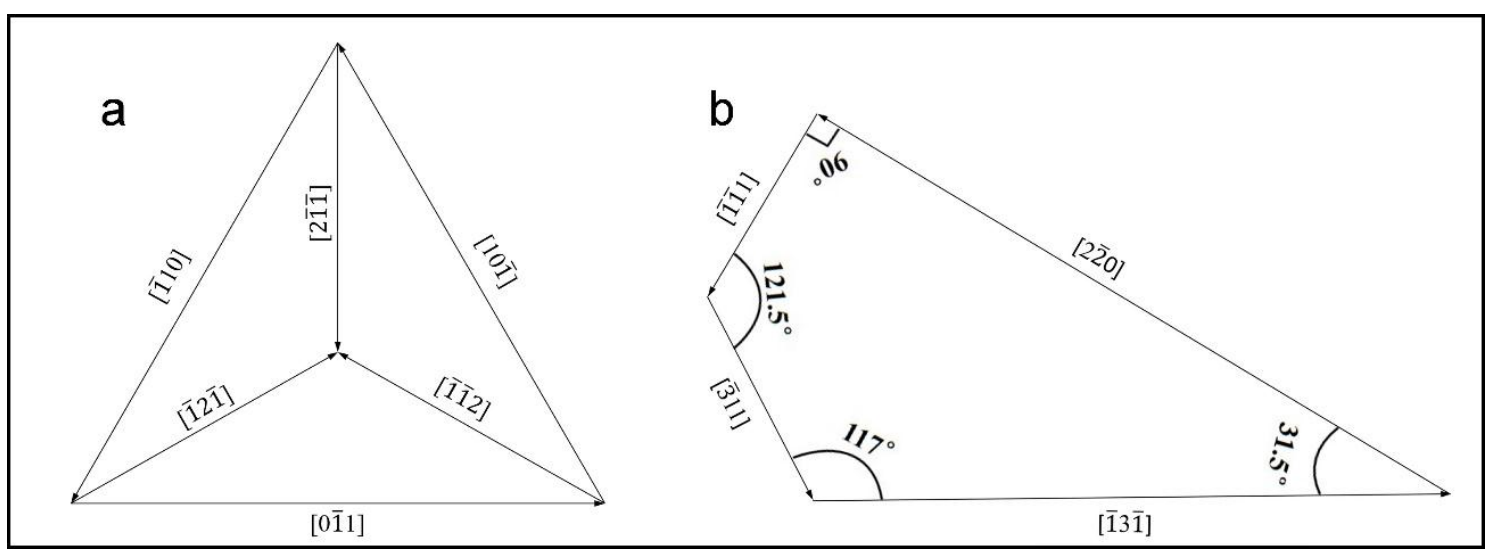

Fig. 2 (a) The geometric model of the (111) etch-pit; (b) the geometric model of the (112) etch-pit.

To further determine the dislocation density, the samples were investigated by synchronization radiation X-ray produced by the Advanced Photon Source (APS) in the II-ID-C high energy X-ray research station of the Argonne National Laboratory. The sample was fixed on the sample platform for rotation or swinging. A beam of high energy X-rays was introduced into the station through a beryllium window-this was 
restricted by a slit that can determine the size of diffraction spots and irradiate the sample. The pattern produced by diffraction was then acquired with a two-dimensional surface detector with a pixel point size of $100 \times 100 \mu \mathrm{m}^{2}$. The incident X-ray was set to 105.1 KeV with a wavelength of $0.11798 \AA$ to produce a diffraction spot as large as $300 \times 300 \mu m^{2}$, when the exposure time was set to 10 s. The high purity $\mathrm{CeO}_{2}$ was used as a standard sample to calibrate the distance between the sample and the detector as well as the azimuth of the detector. The diffraction spots were segmented with Fit2D software. The integral for the first order diffraction spot of $\{110\}$ plane was calculated in order to gain a one-dimensional diffraction pattern. Because the X-ray used in the research station is monochromatic light, the way to analyze the data resembles the way used in conventional X-ray diffraction.

\section{Results and Discussion}

\subsection{Growth habit}

The W coatings deposited on Mo single crystal substrates with $<111>$ axis orientation by CVTD at different temperatures are shown in Fig. 3. 


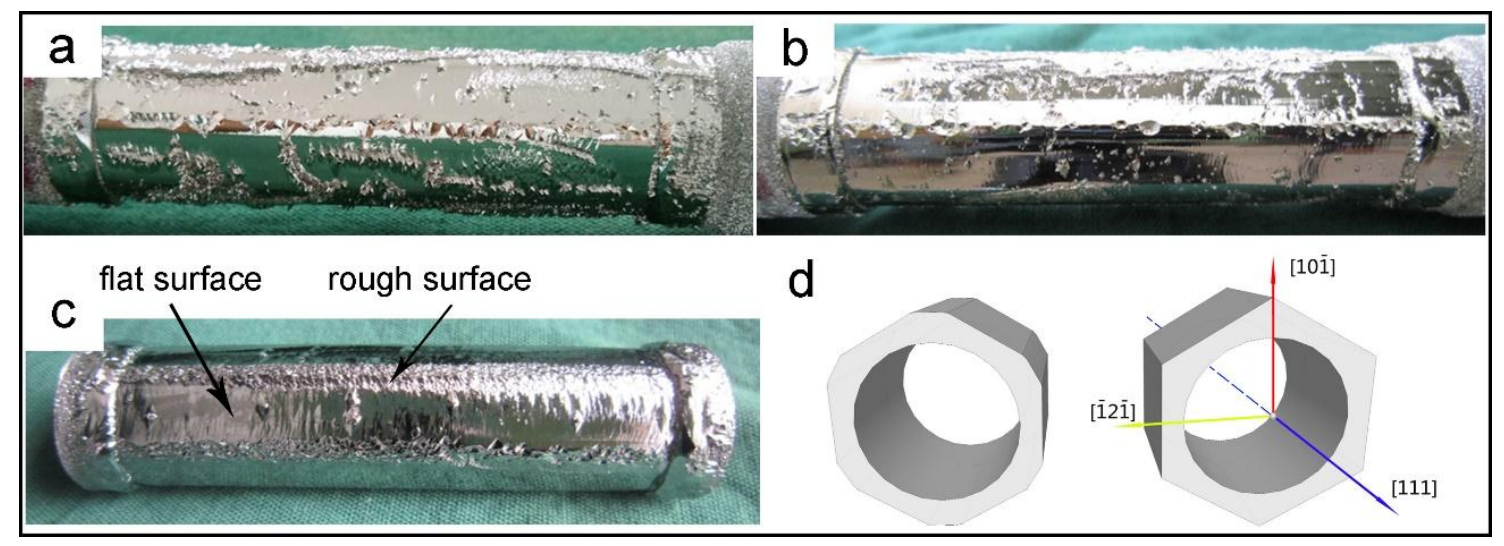

Fig. 3 The macroscopic morphology of the W coatings, (a) W1300, (b) W1300-1400, (c) W1400, and (d) the crystal orientations of the coatings deposited on Mo single crystal substrates with <111> axis orientation.

These coatings exhibit a mirror-like bright surface and are hexagonal prisms. The surface profile contains alternating rough surfaces perpendicular to $\langle 110\rangle$ and flat surfaces perpendicular to $\langle 112\rangle$. The orientations are determined with Laue electron backscattered diffraction. Fig. 3d shows the crystal orientations of the coatings.

According to the works of N.A. Pangarov and G. Weise, the growth rate of two-dimensional nucleus varies with orientations [21, 22]:

Growth rate: $v 111<v 112<v 100<v 110$

Since the growth rate of the $\langle 110\rangle$ orientation is higher than that of the $\langle 112\rangle$, the rough surfaces tend to have greater radial thickness, and smaller area (Fig. 3d left). 
When given enough time, the rough surfaces will develop into the lateral edges (Fig. 3d right).

Fig. 4 shows the microscopic morphology of the W coatings. The rough surface consists of alternating hill-like bulges and pits, while the flat surface exhibits arc-shaped terraces. The formation of this morphology can be explained by the two-dimensional nucleation-mediated lateral growth model $[19,20]$.

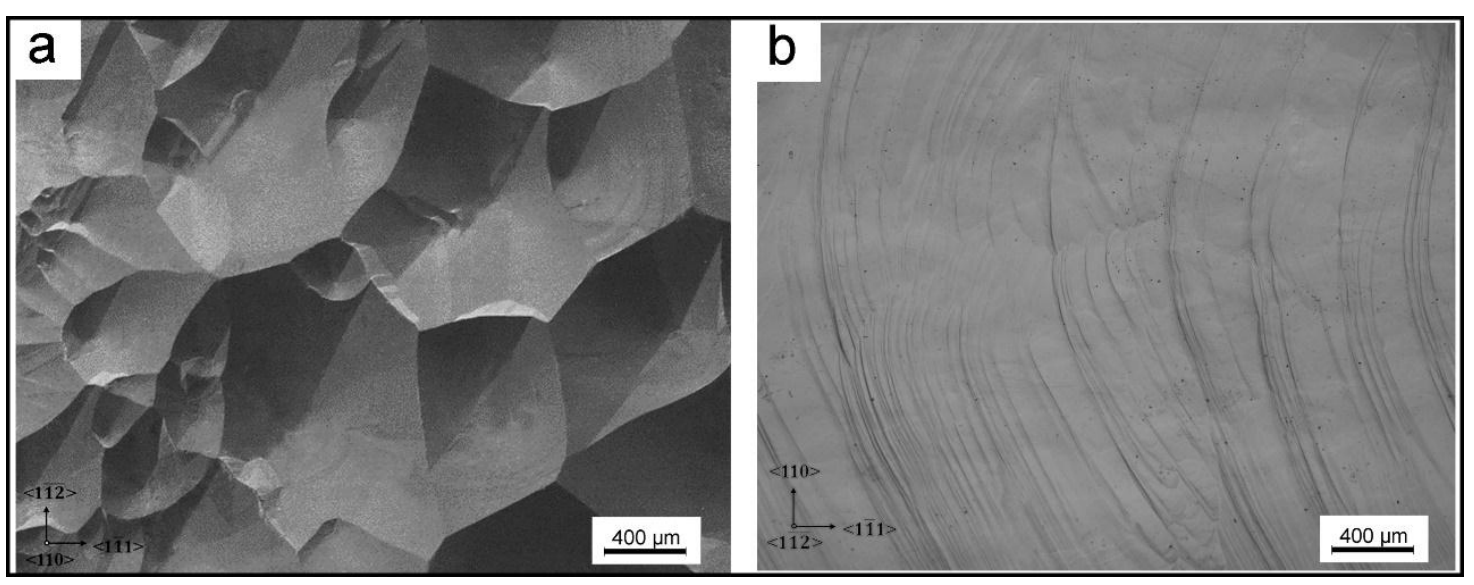

Fig. 4 The typical morphology of the as-deposited W coatings, (a) rough surfaces and (b) flat surfaces.

Because of the growth rate difference mentioned above, on the rough surface, the growth rate of the $\langle 110\rangle$ orientation - which is perpendicular to the surface - is higher. Thus, hill-like bulges and pits are formed on the rough surface (see Fig. 5a). On the flat 
surface, the $\langle 110\rangle$ orientation is parallel to the surface, making the lateral growth faster (see Fig. 5b). This forms the arc-shaped terraces.

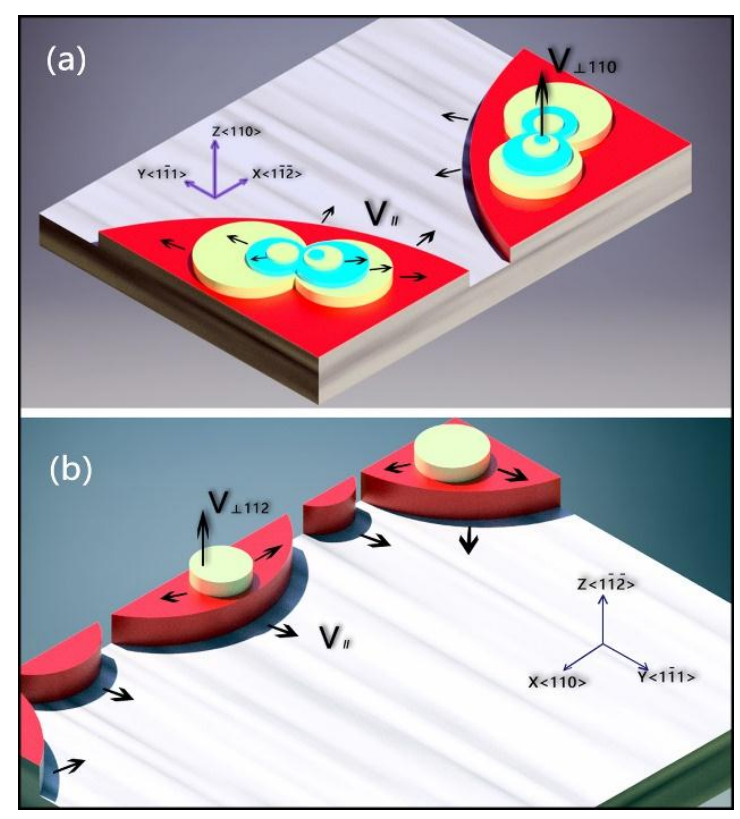

Fig. 5 The schematic diagram of the growth of two-dimensional W nuclei on the substrate, (a) rough surfaces and (b) flat surfaces.

According to crystal growth thermodynamics, the tungsten close-packed $\{110\}$ plane should act as an equilibrium forming plane as a result of its lowest surface energy and should be preserved during the vapor-phase growth process [22]. The facets forming both the rough surfaces and the flat surfaces are therefore $\{110\}$ plane.

\subsection{Abnormal growth}

In the actual deposition process, the morphology of the W coatings can be affected by 
the defects of the substrates and the process parameters including the temperature field and the fluid field of the system. Here, despite the two-dimensional nucleation-mediated lateral growth model mentioned earlier, hillocks of an exotic morphology are also spotted in parts of the coatings (see Fig. 6).

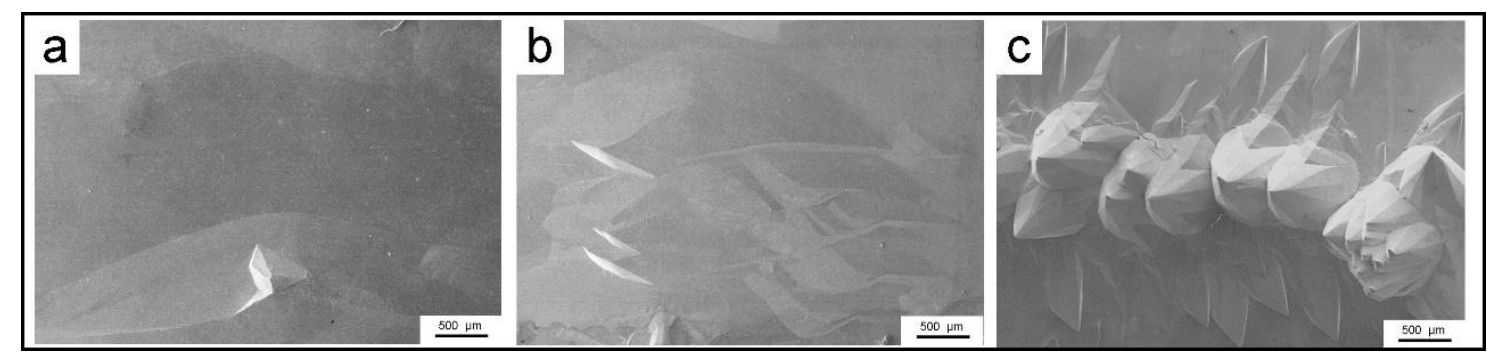

Fig. 6 (a) Single hillock, (b) hillock group, and (c) zonal hillock group with spiny bulges.

Fig. 6a is a micrograph of a single hillock. A cross-sectional area of this hillock was analyzed by EBSD. Fig. 7 shows that in the scanned area, there are no significant color differences (see Fig. 7a). The low-angle boundaries of $2 \sim 15^{\circ}$ account for $100 \%$ (see Fig. 7b) suggesting that the single hillock is likely caused by minor substrate defects like a dislocation outcrop The dislocation outcrop that causes lattice distortion results in a higher energy state in the surrounding area. This can facilitate $\mathrm{W}$ nucleation sites. The two-dimensional nucleus formed on this site grows faster in the orientation perpendicular to the surface - this forms the single hillock on the surface. Since there are no large-angle boundaries, the $\mathrm{W}$ coating is a single crystal. 


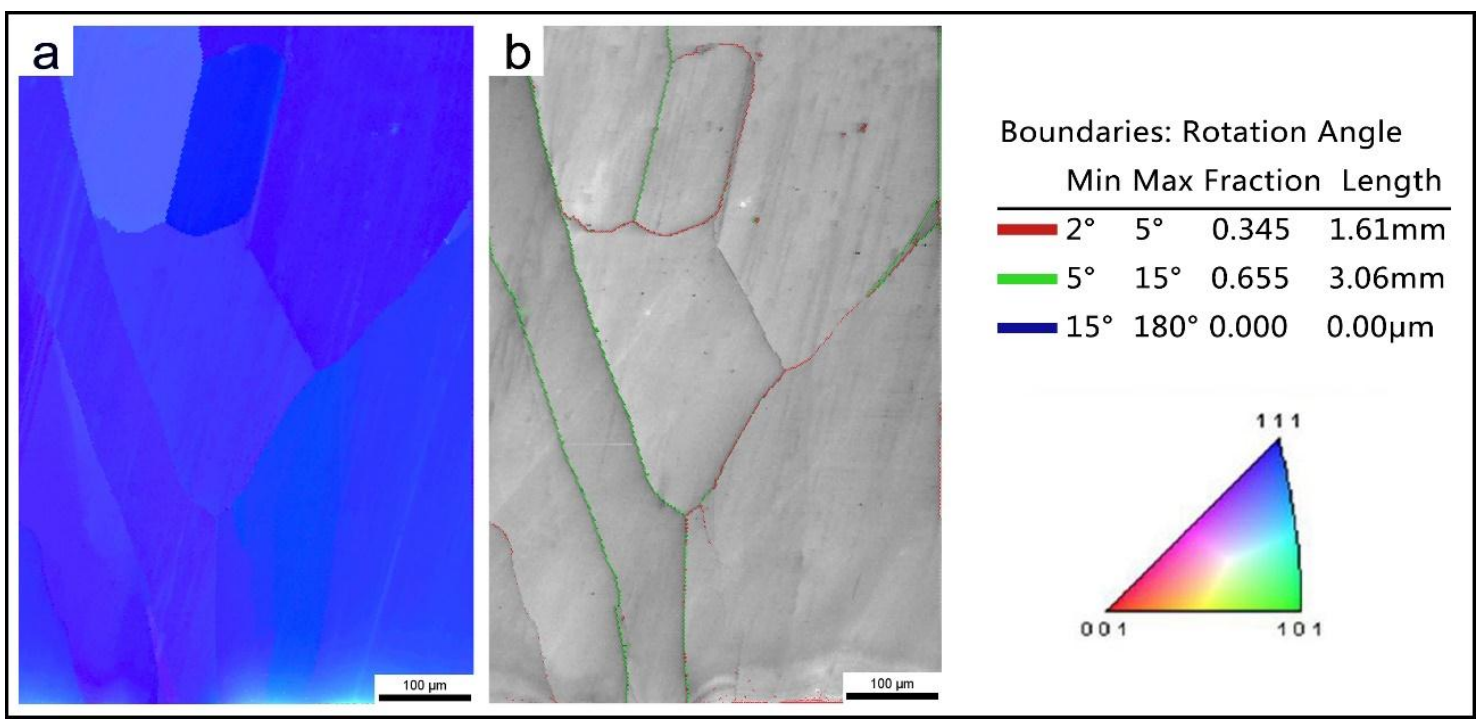

Fig. 7 The cross-sectional area of the single hillock analyzed by EBSD.

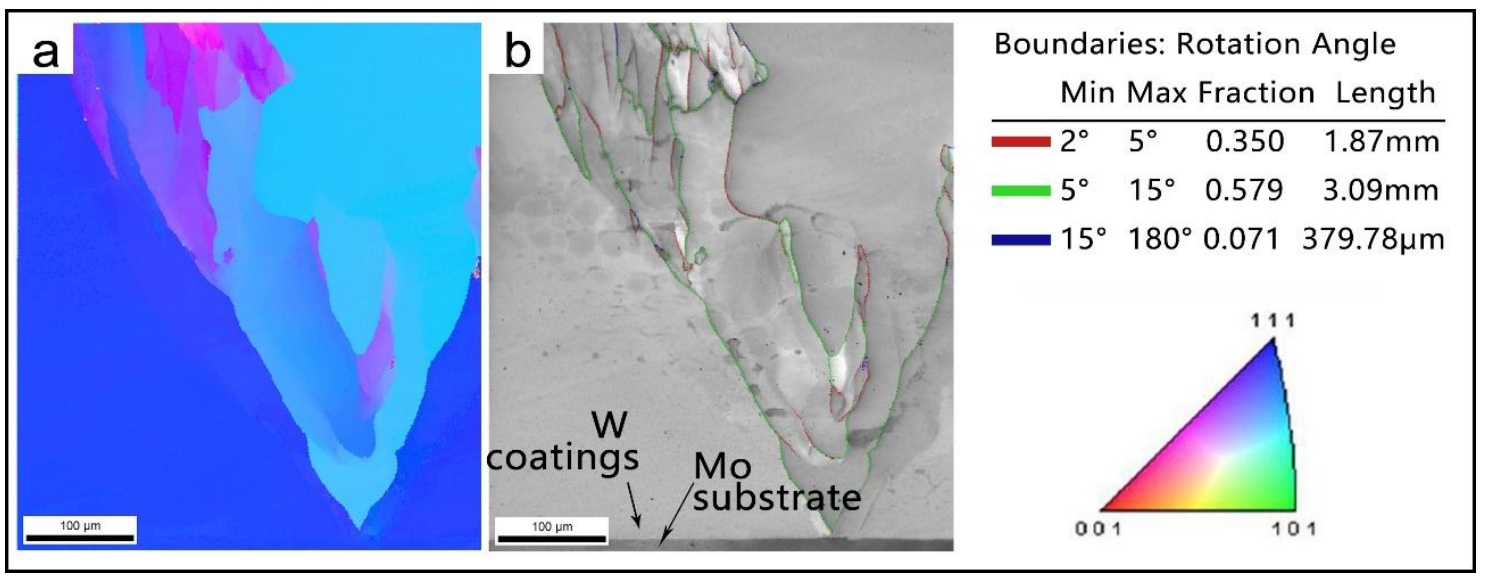

Fig. 8 (a) The cross-sectional area of the hillock group analyzed by EBSD; (b) the EBSD grain orientation map of the group's surface area.

The EBSD grain orientation map of a hillock group (shown in Fig. 6b) is shown in Fig.

8 ( $8 \mathrm{a}$ and $8 \mathrm{~b}$ are from the cross-sectional area). Significant color differences do exist 
(see Fig. 8a) in the cross-sectional area. Low-angle boundaries of $2 \sim 15^{\circ}$ account for $92.9 \%$, and large-angle boundaries of $15 \sim 180^{\circ}$ account for $7.1 \%$ (see Fig. 8b). Obviously, there are sub-boundaries and even boundaries that originate from the Mo substrate in the cross-sectional area of the hillock group.

Fig. 6c shows the morphology of a zonal hillock group with spiny bulges that are significantly different from the morphologies of Fig. 6a and Fig. 6b. Fig. 9 resembles the result of the hillock group and shows that the zonal hillock group also originates at the surface of the substrate where a sub-boundary of higher orientation differences may exist. Sub-boundaries on the surface of the substrate can provide a line of advantageous nucleation sites and gradually form the zonal hillock group. The orientation difference increases as the growth thickness increases and eventually a boundary is formed (see the arrow in Fig. 9).

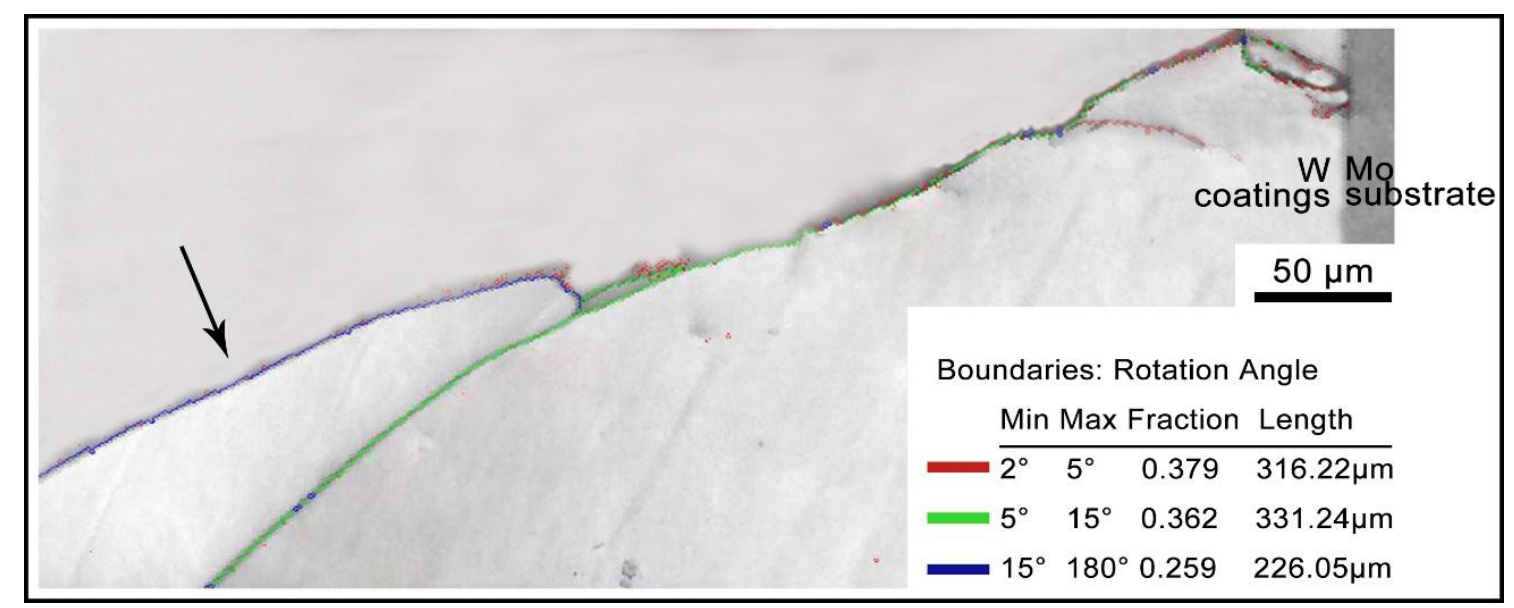

Fig. 9 The cross-sectional area of the zonal hillock group analyzed by EBSD. 
After chemical mechanical polishing with $0.05 \mu \mathrm{m} \mathrm{SiO} 2+\mathrm{H}_{2} \mathrm{O}_{2}$, the same area is observed under $\mathrm{OM}$ where distinct boundaries can be seen with grains in zonal distribution. After electrolytic etching $\left(5 \% \mathrm{NaOH}, 1.5 \mathrm{~A} / \mathrm{cm}^{2}\right)$, different etching morphologies are revealed (see Fig. 10b). According to the theoretical graphs of plane structures given by Thompson [23], different planes have different etching morphologies. In the selected area, grains of different orientations can be seen in Fig. 10a, and sub-boundaries formed by dislocations are marked with arrows.

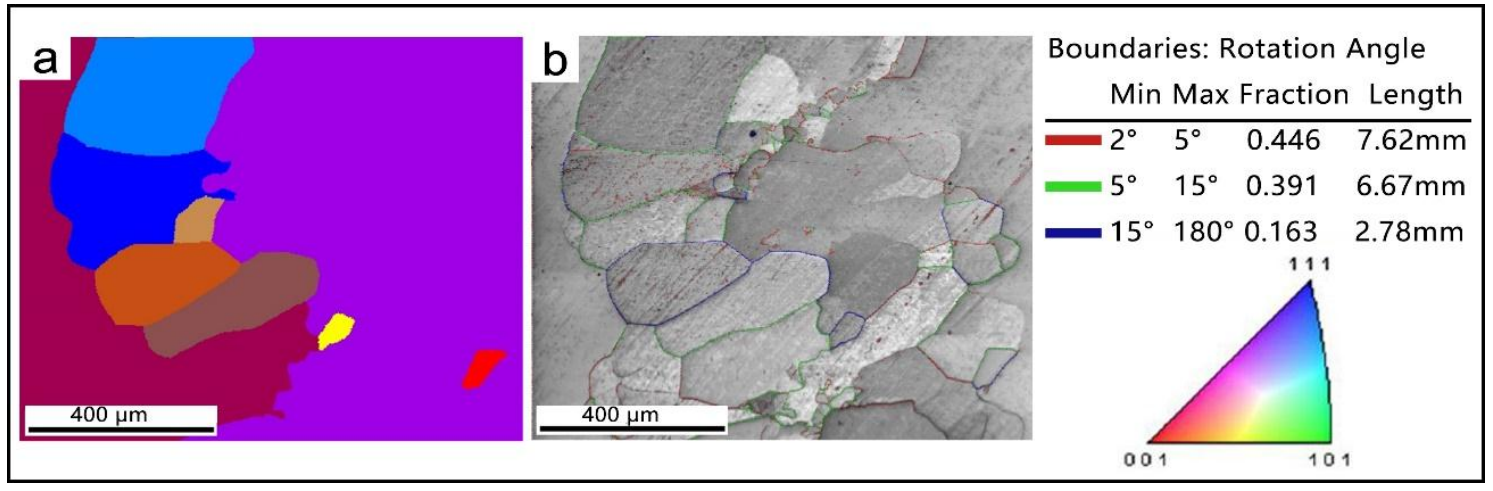

Fig. 10 The EBSD analysis of the zonal hillock group.

In Fig. 10, large-angle boundaries account for the $16.3 \%$ of the total boundaries, and obvious color differences prove that these spiny structures are grains.

In summary, the single hillock, hillock group, and zonal hillock group with spiny bulges are three types of abnormal growth found on the $\mathrm{W}$ coatings. They are likely to be 
caused by the dislocations and sub-boundaries of the Mo substrates. When a single hillock appears, it suggests that the adjacent area contains sub-boundaries, and the coating is a single crystal. However, when more hillocks form a group-especially a group with spiny bulges on them - then boundaries can be expected, and the coating is polycrystalline.

\subsection{Dislocations and sub-boundaries}

Depositing $\mathrm{W}$ on molybdenum substrates is an epitaxial growth process. Thus, the Mo substrates have a significant influence on the quality of the $\mathrm{W}$ coatings. Table 1 shows that the differences in the coefficient of expansion and lattice constant between $\mathrm{W}$ and Mo can lead to lattice misfit and thermal stress, respectively, causing dislocations in the W coatings. On the other hand, during the deposition process, temperature fluctuation can lead to fluctuations in the growth rate. Therefore, foreign atoms can become the nuclei of heterogeneous nucleation and cause multiplication of dislocations.

Fig. 11 shows the micrographs of etch-pits of different planes after being etched. Panels a1, a2, and a 3 are the micrographs of $\{110\}$ planes prepared at different temperatures, and b1, b2, and b3 are $\{112\}$ planes. Panels c1, c2, and c3 are $\{111\}$ planes. Despite the different shapes of the etch-pits, it is obvious that the density of the etch-pits (EPD) decreases and the etch-pits become more distinct from each other as the temperature 
increases (see Fig. 11).

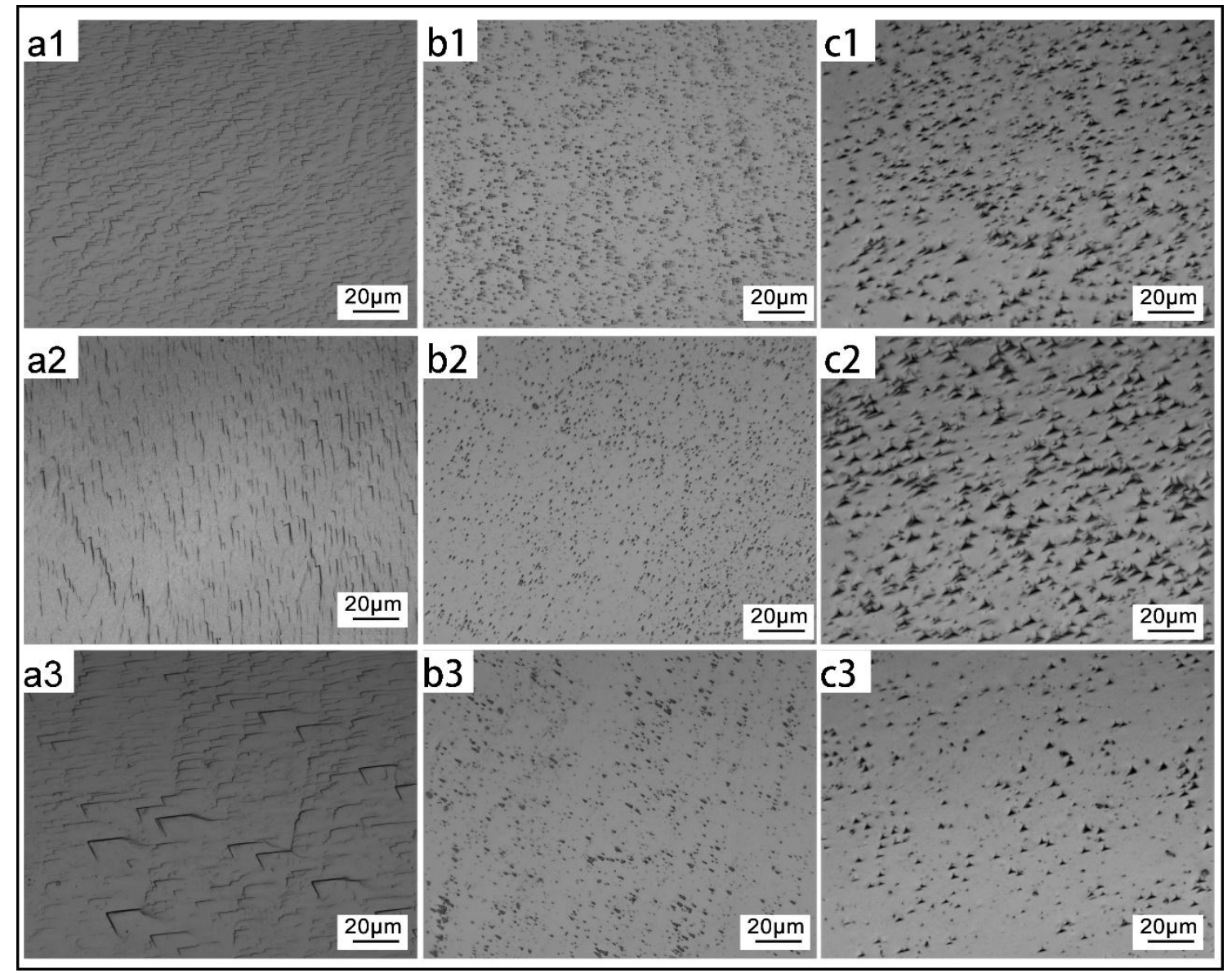

Fig. 11 (a1): $\{110\}$ plane, W1300; (a2): $\{110\}$ plane, W1300-1400; (a3): $\{110\}$ plane, W1400; (b1): $\{112\}$ plane, W1300; (b2): $\{112\}$ plane, W1300-1400; (b3): $\{112\}$ plane, W1400; (c1): $\{111\}$ plane, W1300; (c2): \{111\} plane, W1300-1400; (c3): \{111\} plane, W1400.

The average EPD is listed in the table below (at least three micrographs were measured to get a reliable average EPD).

Table 2. The EPD of different planes from different samples. 


\section{Samples}

$\mathrm{W}_{1300}$

$\mathrm{W}_{1300-1400}$

$\mathrm{W}_{1400}$
EPD (counts/cm²)

$\{110\}$

$8.88 \times 10^{6}$

$2.82 \times 10^{6}$

$2.13 \times 10^{6}$
$\{112\}$

$14.6 \times 10^{6}$

$13.2 \times 10^{6}$

$9.26 \times 10^{6}$
$\{111\}$

$16.5 \times 10^{6}$

$14.6 \times 10^{6}$

$10.2 \times 10^{6}$

As shown in Table 2, The W coatings deposited on the Mo substrates with a dislocation density of $10^{6}$ (counts $/ \mathrm{cm}^{2}$ ) present a dislocation density of $10^{6}$ to $10^{7}$ (counts $/ \mathrm{cm}^{2}$ ). The dislocation density of the $\{110\}$ plane is relatively low. This is because of the two-dimensional multi-nucleation mechanism that allows the $\mathrm{W}$ atoms to form multiple nuclei on the surface of the Mo substrate [24]. Because the growth rate of the nuclei on the $\{110\}$ plane is relatively slow, fewer defects (dislocations) form when two adjacent nuclei come into contact. This results in a lower dislocation density. The high temperature allows the atoms to diffuse to the equilibrium position and allows the dislocations to climb and cross-slip. As a result, the dislocation and internal stress are lower.

Meanwhile, sub-boundaries were spotted on the $\{112\}$ plane and the $\{110\}$ plane of sample $\mathrm{W}_{1300}$ (see the arrows in Fig. 12). Sub-boundaries in Fig. 12 are distinct and of significant length. Fig. 12c shows that the sub-boundaries are formed by coterminous dislocations. The sub-boundaries can be the result of seed crystal inheritance because the Mo substrates used in the experiment contain sub-boundaries. This is due to the repulsion between dislocations that forms an ordered arrangement of dislocations. No 
sub-boundaries were found in $\mathrm{W}_{1300-1400}$ and $\mathrm{W}_{1400}$.

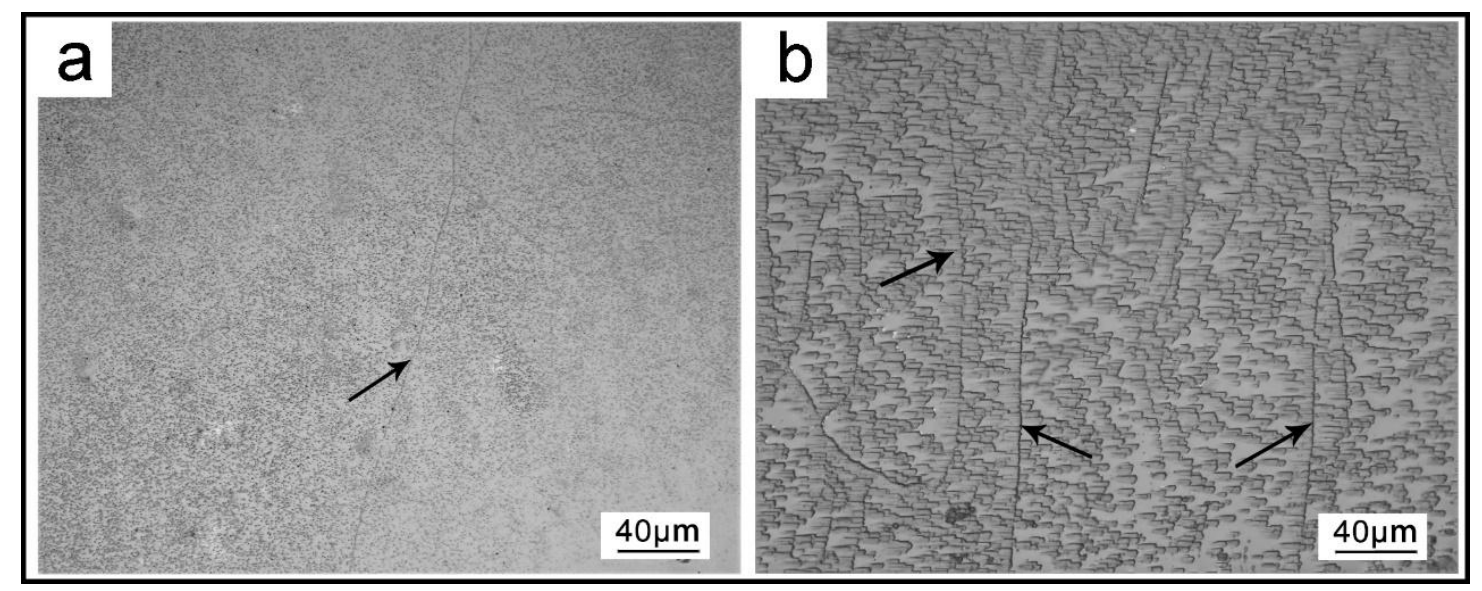

Fig. 12 Sub-boundaries spotted, (a) the $\{112\}$ plane of sample W1300 and (b) the $\{110\}$ plane of sample W1300.

To further determine the dislocation density, synchronization radiation X-ray produced by the APS was applied to the $\{111\}$ planes of different samples- the diffraction pattern of $\mathrm{W}_{1300}$ is shown in Fig. 13a. By using Fit2D to calibrate the diffraction spots, we made sure that the high-energy X-ray irradiated the sample along the $<111>$ axis. Fig. $13 \mathrm{~b}$ is the one-dimensional diffraction curve of the (011) plane diffraction spot of $\mathrm{W}_{1300 \text {. The }}$ full width at half maximum (FWHM) values of different samples are listed in Table 3. These were obtained by applying a Gaussian function to fit the curve. 


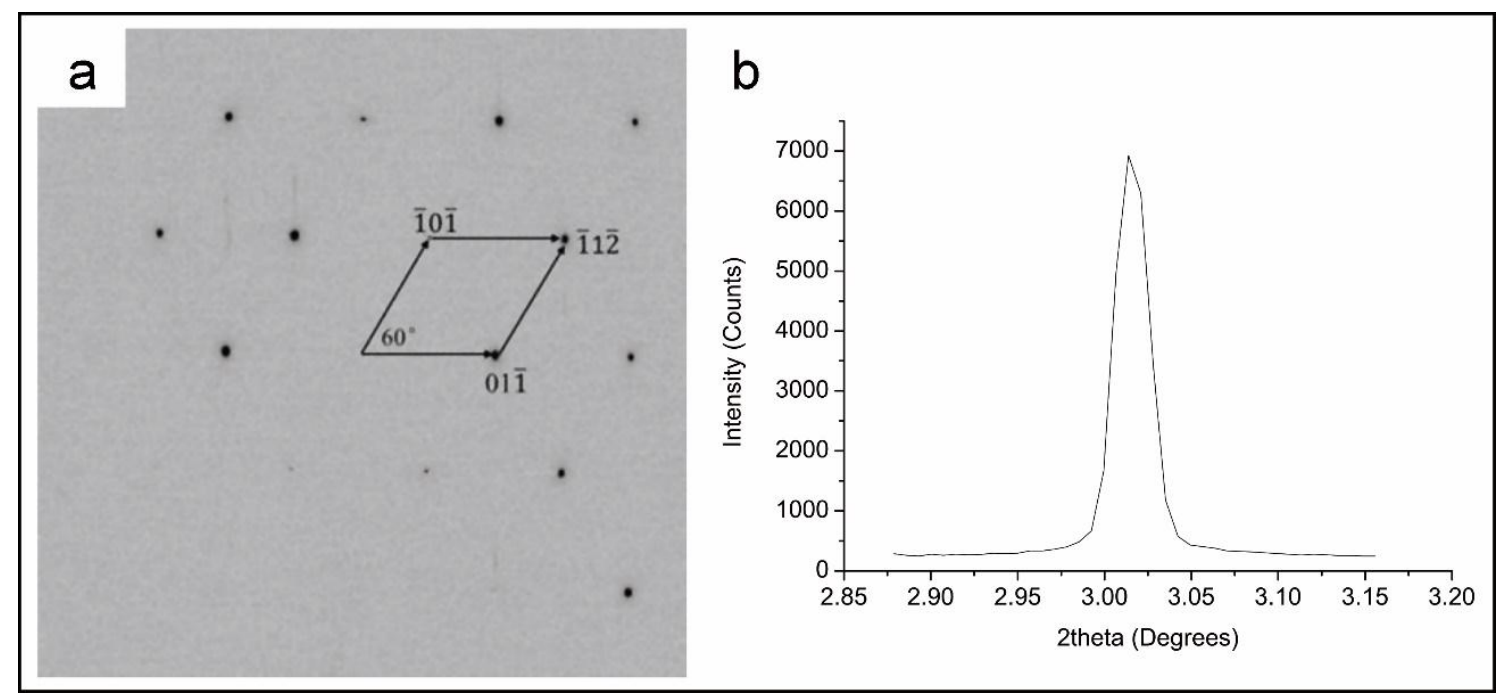

Fig. 13 (a) The synchronization radiation X-ray diffraction patterns of W1300 $\{111\}$ planes; (b) the one-dimensional diffraction curve of the $(01 \overline{1})$ plane synchronization radiation X-ray diffraction spot.

Table 3. The FWHM of different samples.

$\begin{array}{lll}\text { Sample } & \text { FWHM (“) } & \text { Dislocation density (counts/cm }{ }^{2} \text { ) } \\ \mathrm{W}_{1300} & 69 & 3.4 \times 10^{7} \\ \mathrm{~W}_{1300-1400} & 62 & 2.8 \times 10^{7} \\ \mathrm{~W}_{1400} & 53 & 2.0 \times 10^{7}\end{array}$

FWHM ( $\left.\beta_{M}\right)$ is determined by the following equation, where $\beta_{i}$ is the intrinsic FWHM of the crystal, $\beta_{\mathrm{a}}$ is the instrumental constant, $\beta_{\mathrm{d}}$ is the FWHM caused by dislocations, and $\beta_{0}$ is the FWHM caused by residual stress, bending deformation and other factors [25]:

$$
\beta_{M}^{2}=\beta_{i}^{2}+\beta_{a}^{2}+\beta_{d}^{2}+\beta_{o}^{2}
$$

The relation between and the dislocation density $(\rho)$ can be described by the following 
formula [25]:

$$
\rho=\beta_{d}^{2} /\left(4.35 b^{2}\right)
$$

Here, $b$ is the absolute value of the Burgers vector. If we omit $\beta_{i}, \beta_{a}$, and $\beta_{o}$, then dislocation density can be calculated (see Table 3).

The results given by synchronization radiation $\mathrm{X}$-ray illustrates that the dislocation density tends to drop as the temperature increases-obviously, this is consistent with the results of the etch-pit method, despite of the difference that the dislocation density measured by synchronization radiation X-ray is larger than the density obtained by the etch-pit method. This is because omitting $\beta_{i}, \beta_{a}$, and $\beta_{o}$ causes the FWHM to be larger than the true value. On the other hand, the etch-pit method can also result in a lower dislocation density for the following reasons: (1) Some pits may overlap each other; (2) The proper etching time is hard to determine. When the etching time is too short, some etch-pits do not have enough time to form. When the etching time is too long, the early-formed etch-pits may be chemically polished; (3) Some small etch-pit may not be included because the number of etch-pits is automatically counted by computer. In the left of Fig. 14, a dot represents an etch-pit counted by the computer, while in the right, some small and unobvious pits are not counted by the computer. This results in a dislocation density lower than the true value. Therefore, the true dislocation density should be within these two results. 


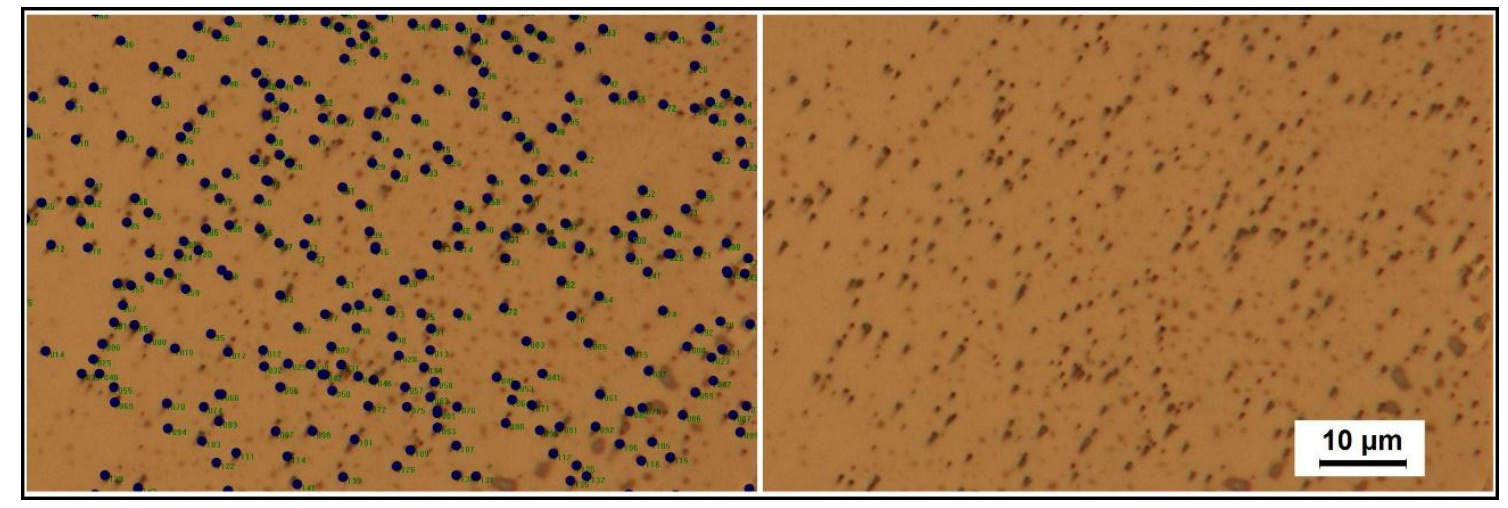

Fig. 14 The etch-pits counted by the computer, (a) counting result, (b) original micrograph of the

$\{112\}$ plane of W1400.

\section{Conclusions}

1. The W coatings achieved by CVTD exhibit a mirror-like bright surface and are hexagonal prisms containing alternating rough surfaces perpendicular to $\langle 110\rangle$ and flat surfaces perpendicular to $\langle 112\rangle$. Different surfaces of $\mathrm{W}$ coatings possess different morphologies. This growth habit can be explained by the the two-dimensional nucleation-mediated lateral growth model.

2. The abnormal growth of $\mathrm{W}$ coatings includes the single hillock, hillock group, and zonal hillock group with spiny bulges. The abnormal growth is likely to be caused by the defects of the Mo substrates. Sub-boundaries but no boundaries are found in the 
adjacent area of the single hillock. Both sub-boundaries and boundaries are found where a group of hillocks appear. When the hillocks have spiny bulges, the formation of boundaries is more severe. Thus, the $\mathrm{W}$ coating is a single crystal when no abnormal growth or only single hillocks appear. Otherwise, it is polycrystalline.

3. The $\{110\}$ plane tends to have the lowest dislocation density. The dislocation density measured by synchrotron X-ray diffraction is larger than that by etch-pit method. Despite this difference, both measurements have a decreasing tendency to dislocate as the temperature increases. Sub-boundaries appearing at $1300^{\circ} \mathrm{C}$, cannot be found in samples with higher depositing temperatures.

\section{Acknowledgements}

This research used resources of the Advanced Photon Source, a U.S. Department of Energy (DOE) Office of Science User Facility operated for the DOE Office of Science by Argonne National Laboratory under Contract No. DE-AC02-06CH11357. This research did not receive any specific grant from funding agencies in the public, commercial, or not-for-profit sectors. 


\section{References}

[1] H.T. Huang, J.P. Zheng, Y.F. Wei, S.J. Chen, Z.D. Wang, C.W. Tan, Microstructure evolution of single crystal tungsten serving under high temperature for long time, Atomic Energy Science and Technology 48S (2014) 528-534.

[2] G.L. Bennett, R.J. Hemler, A. Schock, Space nuclear power - An overview, J. Propul. Power 12 (1996) 901-910.

[3] I.S. Chang, M.H. Hon, Growth characteristics and electrical resistively of chemical vapor deposited tungsten film, Thin Solid Films 333 (1998) 108-113.

[4] V.P. Kobyakov, Promising variants of single crystal tungsten electrodes for high-performance thermionic converters, Tech. Phys. 47 (2002) 1310-1315.

[5] V.G. Glebovsky, V.N. Semenov, The perfection of tungsten single crystals grown from the melt and solid state, Vacuum 53S (1999) 71-74.

[6] R. Cortenraad, S.N. Ermolov, V.N. Semenov, Growth, characterization and surface cleaning procedures for high-purity tungsten single crystals, J. Cryst. Growth 222 (2001) 154-162. 
[7] S.I. Bozhko, V.G. Glebovsky, V.N. Semenov, I. Smirnova, On the growth of tungsten single crystals of high structural quality, J. Cryst. Growth 311 (2008) 1-6.

[8] K.K. Lai, H.H. Lamb, Tungsten chemical vapor deposition using tungsten hexacarbonyl: Microstructure of as-deposited and annealed films, Thin Solid Films 370 (2000) 114-121.

[9] D.C. Mancini, P. Skytt, J. Nordgren, P. Tägtström, Chemical vapour deposition of Cr, Mo and W thin films induced by synchrotron radiation, Vacuum 46 (1995) 1165-1169.

[10] X.N. Ren, H. Zhou, C.C. Ge, X.Q. Liu, Y. Li, W.P. Zhou, W.L. Liu, Z.J. Zhou, X.G. Zhang, M. Xia, Proposal and research on using tungsten carbonyl-CVD process for making W-coating PFMs and W-tubes, Journal of Nuclear Materials 455 (2014) $582-585$.

[11] K.L. Bjorklund, C. Ribbing, H. Norde, M. Boman, Containerless fabrication of tungsten single crystals using laser CVD for field emission applications, Appl. Phys. A 75 (2002) 493-496. 
[12] M. Binnewies, R. Glaum, M. Schmidt, P. Schmidt, Chemical Vapor Transport Reactions, De Gruyter, Berlin, 2012.

[13] Y.W. Lv, X.D. Yu, C.W. Tan, H.L. Ma, J.P. Zheng, F.C. Wang, H.N. Cai, Deposition temperature effects on tungsten single-crystal layer by chemical vapor transport, J. Cryst. Growth 329 (2011) 62-66.

[14] Y.W. Lv, X.D. Yu, F.C. Wang, C.W. Tan, Q.F. Yang, J.P. Zheng, Z.D. Wang, H.N. Cai, Vapor phase epitaxy of monocrystal tungsten coatings, J. Cryst. Growth 387 (2014) 111-116.

[15] R.D. Mu, C.W. Tan, X.D. Yu, Proportion quantitative analysis and etching of $\{110\}$ planes on tungsten single crystal coating surface, J. Alloys Compd. 666 (2016) 71-76.

[16] L. Zhang, Y.L. Shao, Y.Z. Wu, X.P. Hao, X.F. Chen, S. Qu, X.G. Xu, Characterization of dislocation etch pits in HVPE-grown GaN using different wet chemical etching methods, J. Alloys Compd. 504 (2010) 186-191.

[17] Q.C. Liao, H.J. Wang, Y. Wang, F.L. Lan, The Quantitative Analysis of Etch-pits and Crystal Orientations of BCC Metal, Acta Metall. Sin. 18 (1982) 713-725. 
[18] J.R. Thompson, J.C. Danko, T.L. Gregory, H.F. Webster, Surface characterization studies on chemically vapor deposited tungsten, IEEE. T. Electron Dev. 16 (1969) 707-712.

[19] A.E.D.M. Van der Heijden, Handbook of Crystal Growth, North-Holland, Amsterdam, 1994.

[20] D.J. Shu, M. Wang, F. Liu, Z.Y. Zhang, R.W. Peng, R. Zhang, N.B. Ming, Nucleation-Mediated Lateral Growth on Foreign Substrate, Journal of Physics Chemistry C 111 (2007) 1071-1075.

[21] N.A. Pangarov, The crystal orientation of electrodeposited metals, Electrochim. Acta 7 (1962) 139-146.

[22] G. Weise, G. Owsian, The Growth of Tungsten Single Crystals from the Gas Phase during Chemical Reactions, Journal of the Less-Common Metals 22 (1970) 99-116.

[23] J.R. Thompson, J.C. Danko, T.L. Gregory, H.F. Webster, Surface characterization studies on chemically vapor deposited tungsten, IEEE. T. Electron Dev. 16 (1969) 
707-712.

[24] I.V. Markov, Crystal Growth for Beginners: Fundamentals of Nucleation, Crystal Growth and Epitaxy, World Scientific, Singapore, 1995.

[25] R.X. Jia, Y.M. Zhang, Y.M. Zhang, H. Guo, Calculation of Dislocation Destiny Using X-Ray Diffraction for 4H-SiC Homoepitaxial Layers, Spectrosc. Spect. Anal. 30 (2010) 1995-1996.

\section{Figure captions:}

Fig. 1 Three typical types of different etch-pit morphologies, (a) $\{110\}$, (b) $\{112\}$ and (c) $\{111\}$.

Fig. 2 (a) The geometric model of the (111) etch-pit; (b) the geometric model of the (112) etch-pit.

Fig. 3 The macroscopic morphology of the $\mathrm{W}$ coatings, (a) $\mathrm{W}_{1300}$, (b) $\mathrm{W}_{1300-1400}$, (c) $\mathrm{W}_{1400}$, and (d) the crystal orientations of the coatings deposited on Mo single crystal 
substrates with $<111>$ axis orientation.

Fig. 4 The typical morphology of the as-deposited W coatings, (a) rough surfaces and (b) flat surfaces.

Fig. 5 The schematic diagram of the growth of two-dimensional $\mathrm{W}$ nuclei on the substrate, (a) rough surfaces and (b) flat surfaces.

Fig. 6 (a) Single hillock, (b) hillock group, and (c) zonal hillock group with spiny bulges.

Fig. 7 The cross-sectional area of the single hillock analyzed by EBSD.

Fig. 8 (a) The cross-sectional area of the hillock group analyzed by EBSD; (b) the EBSD grain orientation map of the group's surface area.

Fig. 9 The cross-sectional area of the zonal hillock group analyzed by EBSD.

Fig. 10 The EBSD analysis of the zonal hillock group. 
Fig. 11 (a1): $\{110\}$ plane, $W_{1300}$; (a2): $\{110\}$ plane, $W_{1300-1400 ;}$ (a3): $\{110\}$ plane, $\mathrm{W}_{1400}$; (b1): $\{112\}$ plane, $\mathrm{W}_{1300} ;(\mathrm{b} 2):\{112\}$ plane, $\mathrm{W}_{1300-1400}$; (b3): $\{112\}$ plane, $\mathrm{W}_{1400}$; (c1): $\{111\}$ plane, $\mathrm{W}_{1300} ;(\mathrm{c} 2):\{111\}$ plane, $\mathrm{W}_{1300-1400} ;(\mathrm{c} 3):\{111\}$ plane, $\mathrm{W}_{1400}$.

Fig. 12 Sub-boundaries spotted, (a) the $\{112\}$ plane of sample $W_{1300}$ and (b) the $\{110\}$ plane of sample $\mathrm{W}_{1300}$.

Fig. 13 (a) The synchronization radiation X-ray diffraction patterns of $W_{1300}\{111\}$ planes; (b) the one-dimensional diffraction curve of the (01) plane synchronization radiation X-ray diffraction spot.

Fig. 14 The etch-pits counted by the computer, (a) counting result, (b) original micrograph of the $\{112\}$ plane of $\mathrm{W}_{1400}$.

\section{Table captions:}

Table 1. Physical properties of $\mathrm{W}$ and Mo.

Table 2. The EPD of different planes from different samples.

Table 3. The FWHM of different samples. 


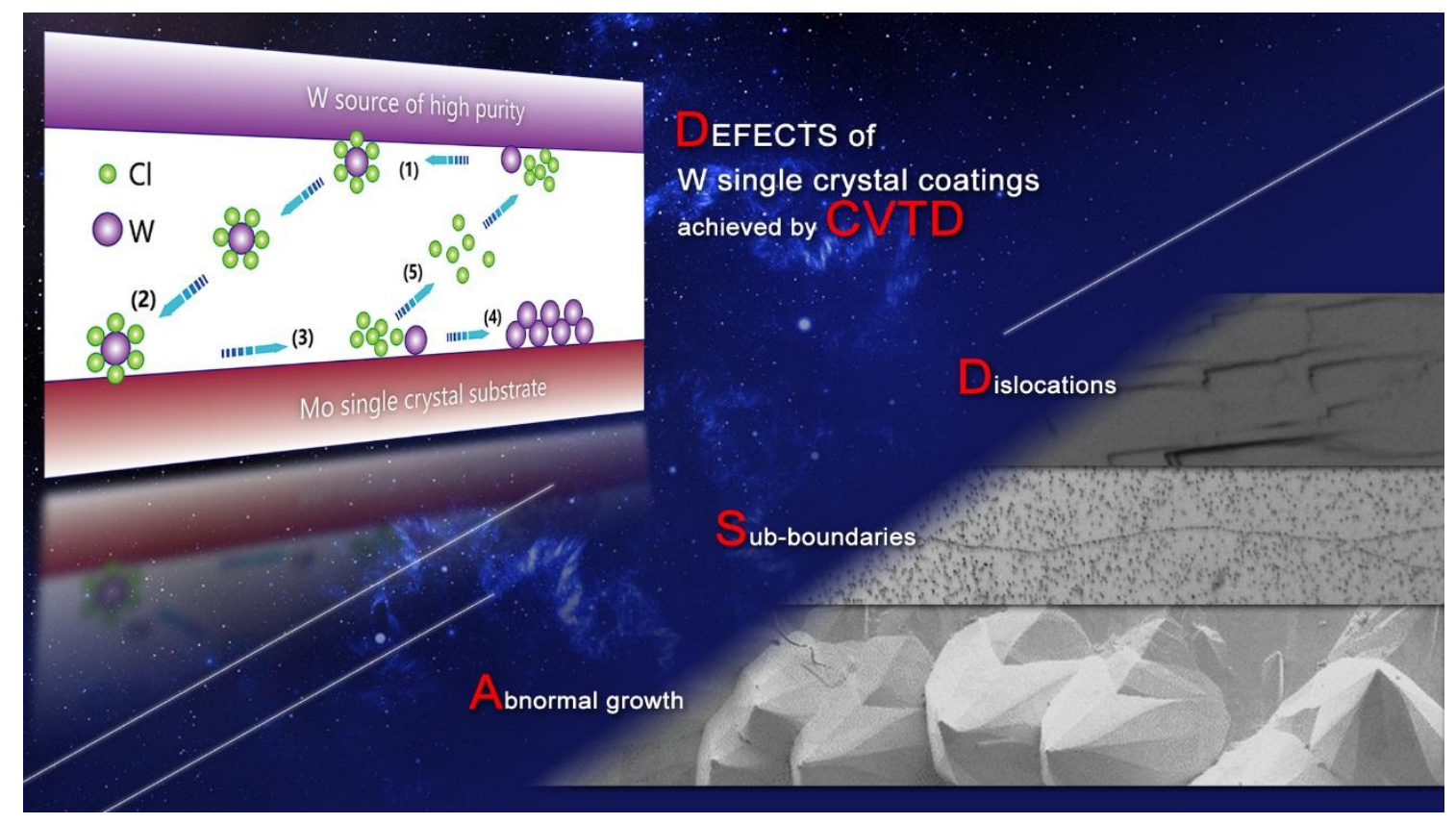

